\title{
Construction and assessment of bio-engineered intervertebral discs
}

\author{
HONGFEI XIANG ${ }^{1 *}$, YAZHOU LIN $^{1 *}$, NANA SHEN $^{2}$, YAN WANG $^{1}$, \\ XIAOLIN WU ${ }^{1}$, GUOQING ZHANG ${ }^{1}$, XUEXIAO MA ${ }^{1}$ and BOHUA CHEN ${ }^{1}$ \\ Departments of ${ }^{1}$ Orthopedic Surgery and ${ }^{2}$ Rehabilitation, \\ The Affiliated Hospital of Qingdao University, Qingdao, Shandong 266003, P.R. China
}

Received March 20, 2016; Accepted February 24, 2017

DOI: $10.3892 / \mathrm{etm} .2017 .4764$

\begin{abstract}
The present study assessed the value of bone marrow-mesenchymal stem cells (BM-MSCs) transformed by nucleus pulposus cells (NPs) for engineering of intervertebral discs. BM-MSCs and fetal NPs were cultured, planted onto polylactic acid-polyglycolic acid co-polymer (PLGA) and observed under inverted and scanning electron microscopes. PLGA scaffolds with adherent or suspended BM-MSCs and NPs were implanted into intervertebral discs of New Zealand white rabbits. Intervertebral signal intensity was evaluated by Thompson grading after 12 weeks. Proteoglycan and type II collagen were measured spectrophotometrically and immunohistochemically, respectively. Spindle or multi-angular BM-MSCs developed fibro-like phenotypesin co-culture with NPs and grew with a normal morphology when attached to PLGA scaffolds. A significant difference was observed in intervertebral proteoglycan expression and collagen II expression in the PLGA scaffold group vs. that in the control group implanted with BM-MSCs and NPs without a scaffold (3.93 \pm 0.31 vs. $3.52 \pm 0.26 \mathrm{mg} / 100 \mathrm{mg}, 12.70 \pm 2.83$ vs. $9.50 \pm 2.06$, respectively). Thus, BM-MSCs can be co-cultured with NPs to enhance their differentiation into NPs for disc regeneration. In conclusion, PLGA scaffolds offer viable growing conditions and allow for the maintenance of mechanical properties and spatial structures of the engineered tissue, which meets the requirements of tissue-engineered discs that do not degenerate.
\end{abstract}

Correspondence to: Dr Bohua Chen or Dr Xuexiao Ma, Department of Orthopedic Surgery, The Affiliated Hospital of Qingdao University, 59 Haier Road, East, Qingdao, Shandong 266003, P.R. China

E-mail: bhchen@hotmail.com

E-mail:ma_xuexiao@126.com

*Contributed equally

Key words: mesenchymal stem cells, tissue engineering, interverbebral disc, polylactic acid-polyglycolic acid copolymer

\section{Introduction}

Approximately $80 \%$ of the aging population suffer from low back pain at some point in their lives $(1,2)$. Back pain is typically associated with intervertebral disc degeneration (IDD) and dramatically affects quality of life, work productivity and significantly impacts health care spending (3). Lower back pain predominantly arises from intervertebral disc degeneration within the cell and its matrix, and it is difficult to reverse (4). Current treatments, including excision of the nucleus pulposus (NP) and intervertebral fusion, do not solve the fundamental problem (5). Thus, engineering materials that restore the structure and function of intervertebral discs are of enormous interest $(5,6)$.

As human bone marrow, mesenchymal stem cells (BM-MSCs) are convenient, lack immunogenicity and have multi-directional differentiation potential, they are commonly used as 'seeds' for biomaterial engineering. Polylactic acid (PLA)-polyglycolic acid (PGA) co-polymer (PLGA), a polymer of glycolide and lactide, is biocompatible and is absorbed by human cells, rendering it an optimal composite biological scaffold that is widely used as an extracellular matrix material (7). The present study used BM-MSCs that had a multi-directional differentiation potential and differentiated into nucleus pulposus cells (NPs). These cells were inoculated into PLGA scaffolds and grown tissue was assessed for defects and utility for engineering tissues that were later tested in animal models. The present study investigated the construction and assessment of bio-engineered intervertebral discs. The results provide fundamental information for engineering materials therapy to decelerate IDD in future clinical application.

\section{Materials and methods}

Materials. Polylactic acid (PLA)-polyglycolic acid (PGA) co-polymer (PLGA) scaffolds were obtained from Jinan Daigang Biomaterial Co., Ltd. (Jinan, China) and included low-temperature, high-pressure PLA (PLA/PGA ratio, 75:25; porosity, $>90 \%$; aperture, 200-250 $\mu \mathrm{m}$; molecular weight, $300,000 \mathrm{kDa}$ ). Transforming growth factor (TGF)- $\beta 1$ was obtained from Peprotech (Rocky Hill, NJ, USA). BM-MSCs with fluorescent markers (third generation, Odcell HUxMA 
late 1101080925A01) were purchased from Guangzhou Cyagen Biotechnology Co., Ltd. (Guangzhou, China). Streptavidin-peroxidase immunohistochemical staining kits (SP-0022) were obtained from Beijing Biosynthesis Biotechnology Co., Ltd (Beijing, China).

$N P$ cultures. Once informed consent was obtained, a total of 3 voluntary donations of aborted human fetuses ( $\sim 35$ weeks of gestation) were used, which was approved by the Ethics Committee of the Affiliated Hospital of Qingdao University Medical College. NPs from fetal tissue were cut away, ground into a paste and then digested with $0.2 \%$ collagenase II (MP Biomedicals, LLC; Santa Ana, CA, USA). This produced a clear liquid $(5 \mathrm{ml})$ that was centrifuged with $5 \mathrm{ml}$ low-glucose Dulbecco's modified Eagle's medium (L-DMEM; GE Healthcare Life Sciences, Chalfont, UK) containing 10\% fetal calf serum (Gibco; Thermo Fisher Scientific, Inc., Waltham, MA, USA). This mixture was placed in an incubator at $37^{\circ} \mathrm{C}$ containing $5 \% \mathrm{CO}_{2}$ in air and cells were grown to $90 \%$ confluence.

$B M-M S C$ recovery and passage. Samples were placed in a warm water bath to thaw and then transferred to a centrifuge tube. L-DMEM was added to the tubes and samples were mixed with gentle pipetting and then centrifuged 3 times. Cells were re-suspended in $4 \mathrm{ml}$ cell culture medium and seeded in culture bottles.

Electron microscopy of PLGA scaffolds and preparation of intervertebral disc tissue engineering constructs. PLGA scaffolds were cut into $4 \times 2-\mathrm{mm}$ blocks, rinsed with saline, dried and sterilized with ethylene oxide. Scaffolds were immersed in L-DMEM, followed by fixing in glutaraldehyde, dehydration with a graded ethanol series and drying. An ion sputtering apparatus was used to electroplate samples in gold for subsequent electron microscopy.

BM-MSCs and NPs were detached by digestion in $0.25 \%$ and mixed at a 1:3 ratio. The resulting cell suspension $\left(1 \times 10^{5}\right.$ cells $\left./ \mathrm{ml}\right)$ was seeded onto the PLGA scaffold and cultured for $1 \mathrm{~h}$. The scaffold was inverted and allowed to incubate for $5 \mathrm{~h}$ in L-DMEM containing $5 \mu \mathrm{g} / 1 \mathrm{TGF}-\beta 1$.

Implantation of engineered intervertebral discs. Male New Zealand white rabbits $(n=20$; age, 6 months; weight, $2.0-2.5 \mathrm{~kg}$ ) were obtained from the Qingdao Institute for Drug Control (Qingdao, China). The rabbits were maintained separately at room temperature, exposed to a $16: 8 \mathrm{~h}$ light: Dark cycle and were fed with rabbit feed (Qingdao Kangda Foodstuffs Co., Ltd., Qingdao, China). The rabbits had free access to water. The rabbits were anesthetized with Su Mian Xin II $(0.15 \mathrm{ml} / \mathrm{kg}$ intramuscularly; Kangda Medical Products Co., Ltd., Shanghai, China) and atropine sulfate $(0.05 \mathrm{mg} / \mathrm{kg}$ intramuscularly; Jiangsu Lianshui Pharmaceutical Co., Ltd., Lianshui, China). Animals were subjected to lumbar magnetic resonance imaging (MRI) and then randomly divided into 2 groups. Each animal received an anterolateral abdomen oblique incision parallel to the iliac crest through an extraperitoneal operative route to expose the L4-5 intervertebral space. Pre-vertebral annuli of control animals were destroyed and the NP was removed. Subsequently, a BM-MSC and NP suspension $\left(1 \times 10^{5}\right.$ cells $/ \mathrm{ml}$; ratio=1:3) was implanted into the intervertebral space $(50 \mu \mathrm{l})$. Animals in the experimental group were treated equally, but PLGA scaffolds $(4 \times 2-\mathrm{mm}$ blocks) with an adherent equivalent of BM-MSCs and NPs were implanted into the intervertebral space. After 12 weeks, another lumbar MRI was performed. Intervertebral signal intensity on MRI was evaluated using a 5-stage Thompson grading scale for describing the gross morphology of the intervertebral discs at 12 weeks after the second MRI was obtained (8). A Thompson grade of 1 indicated normal signal expression in intervertebral discs. For grade 2, the signal was mildly impaired and had a narrow signal range. A Thompson grade of 3 referred to weakened signals and grade- 4 discs showed impaired signaling. Subsequent to MRI, animals were sacrificed. The L4-5 intervertebral discs were removed, frozen, sectioned $(6 \mu \mathrm{m})$ and histochemically assessed for differences between control and experimental animals.

Detection of protein polysaccharide and collagen type II. At 12 weeks after the operation, the expression of aggrecan in the intervertebral discs was detected by benzene-3-phenol spectrophotometric detection used to represent protein polysaccharide (9). For detection of collagen II, 10\% paraformaldehyde-fixed intervertebral disc tissue were embedded in paraffin. Paraffin sections were baked in a $60^{\circ} \mathrm{C}$ oven (Fuzhou Maixin Biotechnology Co., Ltd., Fuzhou, China) for $2 \mathrm{~h}$, deparaffinized with xylene, rehydrated in descending alcohol and then rinsed with sterile distilled water for 3-5 min. Antigen retrieval in tissue sections was performed by using a modified method of microwave antigen retrieval. Tissue sections were cooled to room temperature and washed by using phosphate buffer saline (PBS) three times for $5 \mathrm{~min}$ each. After PBS flushing, the fixed intervertebral disc tissues were incubated for $2 \mathrm{~h}$ at $37^{\circ} \mathrm{C}$ with anti-human collagen type II antibody (1:100 dilution; cat. no. ab3092; Abcam Ltd., Cambridge, MA, USA). Samples were then incubated for $1 \mathrm{~h}$ at room temperature with goat anti-mouse horseradish peroxidase-conjugated secondary antibodies (1:1,000 dilution; cat. no. CW0102S; CWBio, Beijing, China), and then rinsed with PBS. Subsequent to visualization with diaminobenzidine and counterstaining with hematoxylin, staining was observed under a microscope.

Statistical analysis. All values were expressed as the mean \pm standard error of the mean. An independent-samples t-test and a Mann-Whitney rank sum test were applied to assess differences between groups. $\mathrm{P}<0.05$ was considered to indicate a statistically significant difference. Statistical analyses were performed using SPSS software for Windows (version 19; International Business Machines, Corp., Armonk, NY, USA).

\section{Results}

Morphological observation of NPs. NPs attached to culture bottles at $24 \mathrm{~h}$ after inoculation and adhered well after 10 days. Under an inverted microscope, chondrocytes or cartilage-like cells were evident with large round or oval nuclei with 2-3 nucleoli, as well as spindle- or multi-angular fiber-like cells with round or ovoid nuclei and acytoplasm extending as pseudopodia (Fig. 1). 


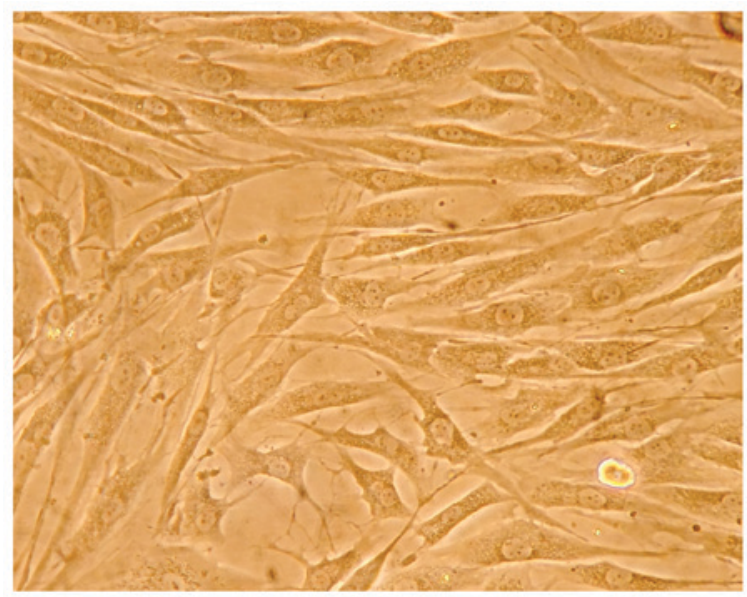

Figure 1. Nucleus pulposus cells were polygonal, round and fusiform, and certain cells were mitotic with a high proliferative capacity as observed under an inverted phase contrast microscope (magnification, $\mathrm{x} 400$ ).

Morphology of BM-MSCs and co-culture of BM-MSCs and NPs. Approximately $95 \%$ of BM-MSCs were round or ovoid and were adherent at $24 \mathrm{~h}$ after recovery. BM-MSCs gradually became spindle or multi-angular shaped over time, and most of them had slim and extended pseudopodia that fluoresced under an inverted fluorescence microscope (Fig. 2). BM-MSCs and NPs adhered and grew well with a vigorous metabolism, and the cell cytoplasm was filled with numerous secretory granules. Each of the two cell types had a spindleor multi-angular fibro-like phenotype in co-culture and was difficult to distinguish from the other based on morphology. Mixed BM-MSCs and NPs grew well and fluorescence was strong (Fig. 3).

Electron-microscopic observation of PLGA scaffolds and comparison of tissue-engineered discs by MRI. Electron microscopy showed that BM-MSCs and NPs grew well with normal morphology when attached to PLGA scaffolds, which suggested that the PLGA scaffolds had an affinity for BM-MSCs and NPs (Fig. 4). A white jelly-like NP (3 mm diameter) was removed via an extraperitoneal approach and BM-MSCs and NPs in suspension were implanted into intervertebral discs of control animals and PLGA scaffolds with adherent BM-MSCs and NPs were implanted in intervertebral discs of the experimental animals. MRI was performed and disk morphology after surgery and 12 weeks' post-surgery was evaluated by Thompson grading: All of rabbits in control and experimental groups $(n=20)$ were Thompson grade 1 and 2 immediately after surgery. After 12 weeks, 3 animals in the control group were Thompson grade 3 and 7 were grade 4 . Furthermore, 2 animals in the experimental group were grade 2, 6 were grade 3 and 2 were grade 4 . Pulposus signal intensity in each group gradually weakened, and the intervertebral space narrowed and degenerated over time. Intervertebral signal intensity in the experimental group was significantly higher than that in controls $(\mathrm{P}=0.015)$. Thus, the recovery of the intervertebral discs in the experimental animals at 12 weeks was superior than that in controls according to Thompson grading (Fig. 5).

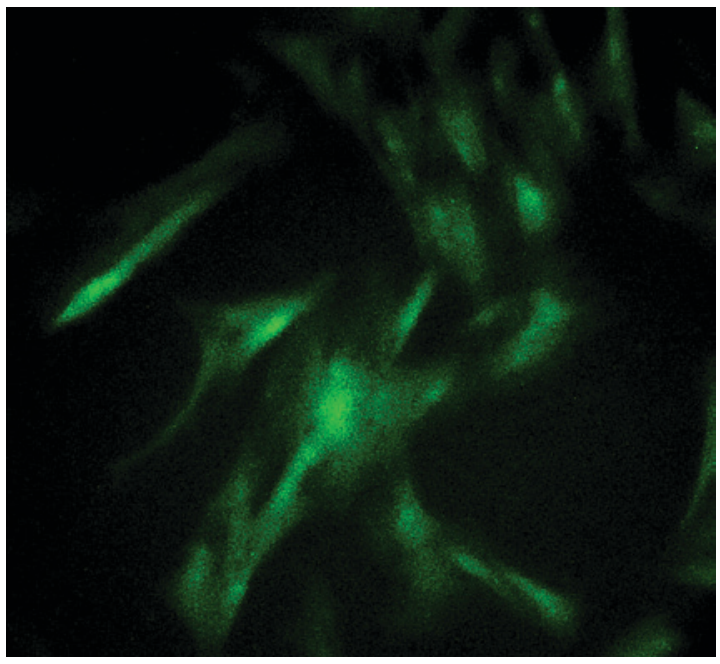

Figure 2. BM-MSCs had extended pseudopodia and were adherent and in an active proliferating state. Cells had fusiform, polygonal and irregular shapes as observed under an inverted phase contrast microscope (magnification, $\mathrm{x} 400$ ). The BM-MSCs contained a large number of fluorescent particles. BM-MSCs, bone marrow-mesenchymal stem cells.

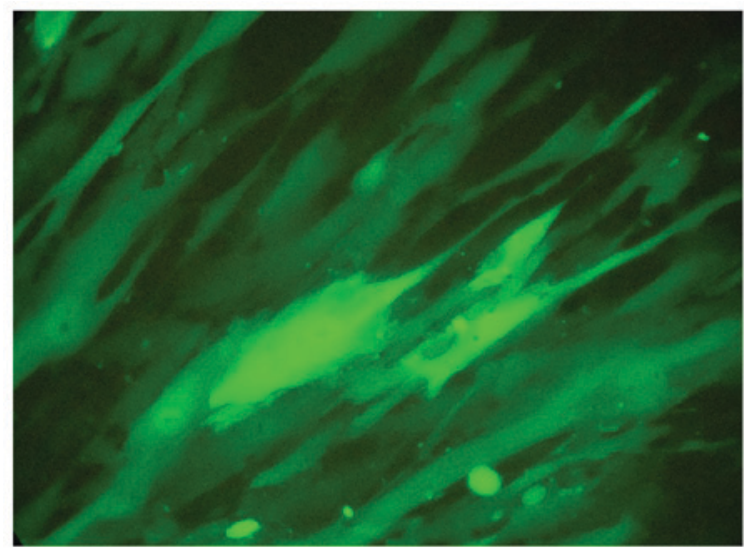

Figure 3. Mixed bone marrow-mesenchymal stem cells and nucleus pulposus cells were adherent and grew well, secreting abundant extracellular matrix that strongly fluoresced under a fluorescent microscope (magnification, x400).

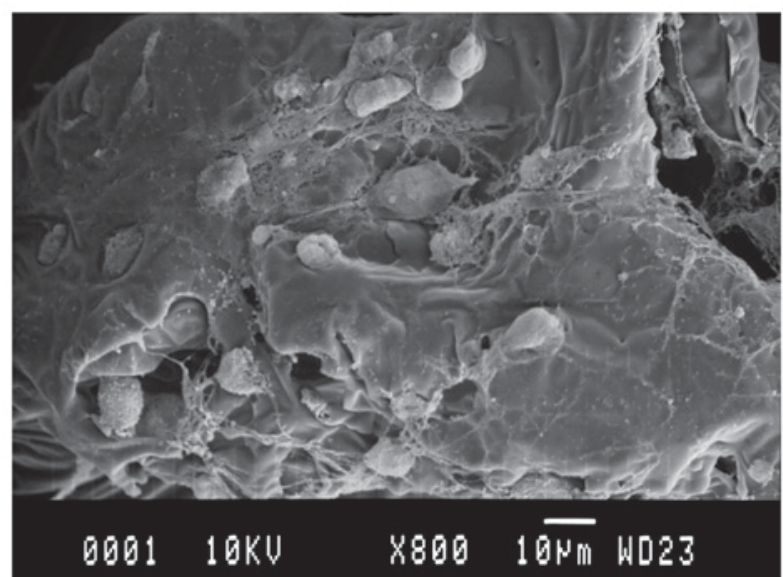

Figure 4. PLGA scaffolds had a uniform fiber mesh aperture as observed under a scanning electron microscope and cells were adherent and grew well, extending pseudopodia and secreting an abundant extracellular matrix when cells were attached and cross-linked to PLGA scaffolds. PLGA, polylactic acid-polyglycolic acid co-polymer (magnification, x800). 

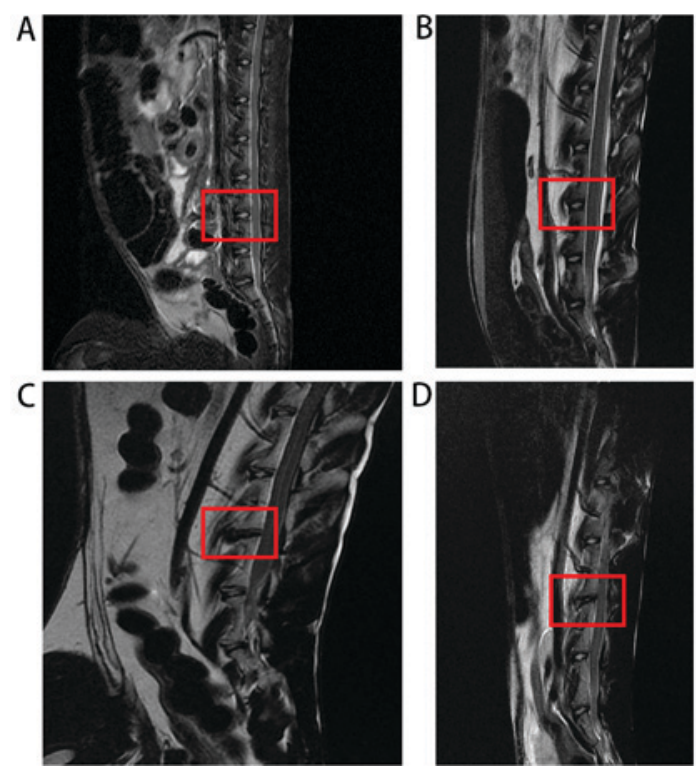

Figure 5. Magnetic resonance imaging detection in the control and experimental group. (A) Control group and (B) experimental group immediately after surgery. (C) Control group and (D) experimental group after 12 weeks. The signal in the experimental group was higher than the control group. Surgery position is marked by the red frame.

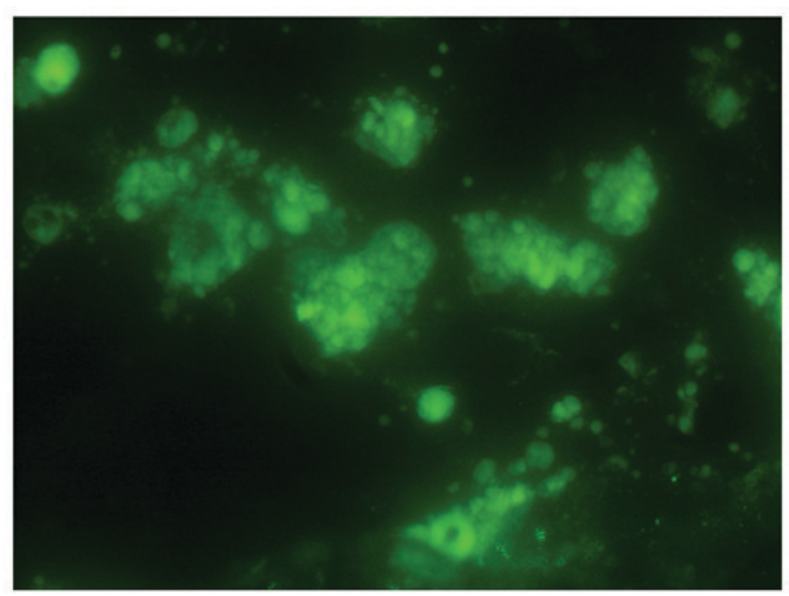

Figure 6. Green fluorescence was observed in intervertebral disc slices 12 weeks after implantation, indicating bone marrow-mesenchymal stem cell survival (magnification, 400).

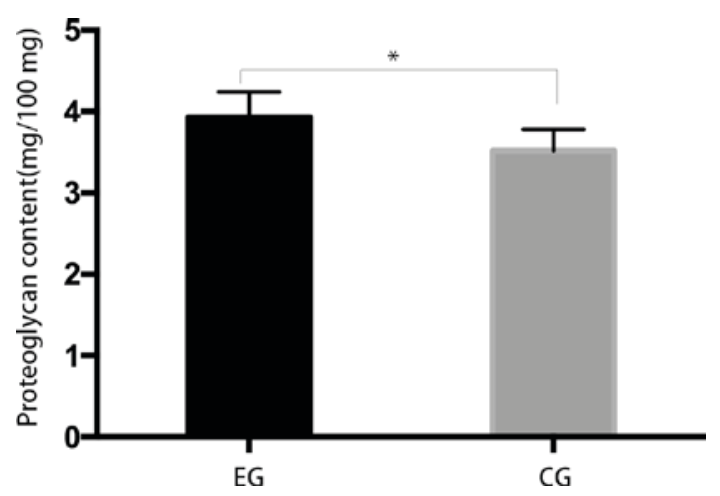

Figure 7. Proteoglycan content were detected by benzene-3-phenol spectrophotometric detection. EG, experimental group; CG, control group. Proteoglycan content of EG was significantly higher than that in the control group. ${ }^{*} \mathrm{P}<0.05$
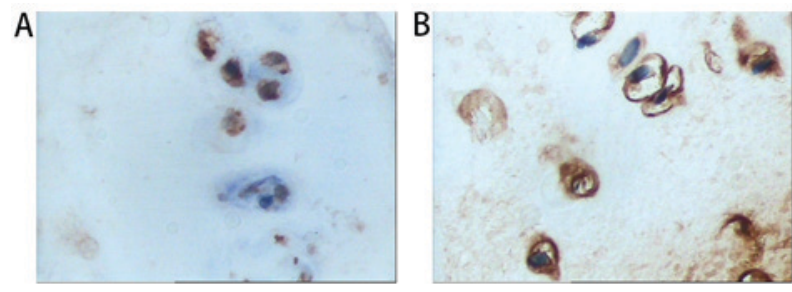

Figure 8. Immunohistochemical detection of collagen II expression in cytoplasts in the (A) control and (B) experimental groups. Immunoreactive cells were brown or pale yellow and cells were counterstained with hematoxylin (magnification, $\mathrm{x} 400$ ).

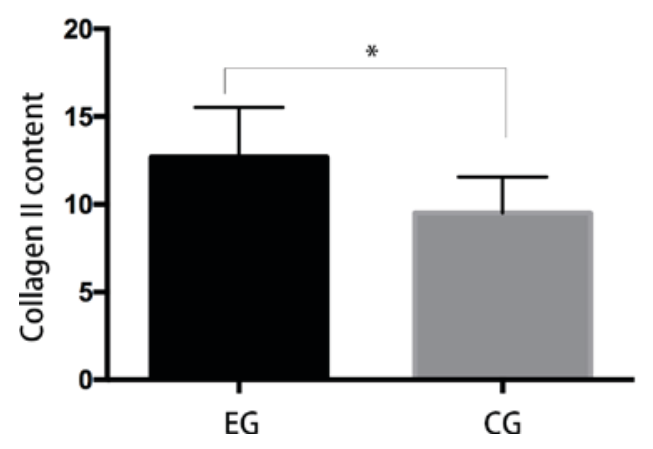

Figure 9. Expression of collagen II was scored according to standard methods. EG, experimental group; CG, control group. Collagen II content was significantly different between experimental and control groups. ${ }^{*} \mathrm{P}<0.05$.

Observations after transplantation and proteoglycan and type II collagen content. Green fluorescence was observed in intervertebral disc slices of the two groups at 12 weeks after implantation, which indicated the presence of live cells within the intervertebral discs (Fig. 6). The proteoglycan content in the experimental group was significantly higher than that in the control group $(3.93 \pm 0.31$ vs. $3.52 \pm 0.26 \mathrm{mg} / 100 \mathrm{mg} ; \mathrm{P}=0.014$; Fig. 7). Type II collagen was measured immunohistochemically. Collagen II-expressing cell cytoplasts in the experimental and control groups stained brown or pale yellow (Fig. 8). The expression was scored according to standard methods. The collagen II content was significantly higher in the experimental group compared with the control group ( $\mathrm{P}=0.012$; Fig. 9).

\section{Discussion}

Regarding their application in the treatment of intervertebral disc degeneration, BM-MSCs have 3 characteristics in common with NPs and annulus fibrosus from autografts, allografts or xenografts $(6,10)$. They provide a cellular energy source and are able to differentiate, while having low immunogenicity (6). BM-MSCs are easy to extract from several adequate available sources (11). Under hypoxia, in the presence of TGFs or in co-culture with NPs, BM-MSCs may transdifferentiate into NPs (12). TGF- $\beta 1$ not only regulates the proliferation of BM-MSCs, but also inhibits inflammation and increases xenograft and allograft transplant safety. Thus, TGF- $\beta 1$ at $5-10 \mu \mathrm{g} / 1$ is the most effective factor for promoting BM-MSC proliferation, differentiation into cartilage and synthesis of extracellular matrix. 
Five-month-old NP cells have been reported to form a visible jelly-like NP and proliferated without degenerating. In addition, notochord cells of chondrocyte nuclei of embryonic discs produced diverse endogenous growth factors, such as TGF- $\beta 1$. This principle was applied by co-culturing NPs and BM-MSCs at a ratio of 3:1 to improve the conversion efficiency of BM-MSCs (12).

In the present study, NPs and BM-MSCs were co-cultured in the presence of TGF- $\beta 1$ and implanted into rabbit intervertebral discs using previously reported methods (13-16). Nerlich et al (17) reported that severe disc defects occurred using the above method, and that blood vessel growth was negligent on the NP. In the present study, only the front of the annulus fibrosus was damaged to reduce interference of tissue surrounding the discs, and the BM-MSCs stimulated lymphocytes without triggering immunoreactivity. Immunosuppressive BM-MSCs have been reported to inhibit $\mathrm{T}$ cells in vivo and in vitro (18). Thus, xenografted BM-MSCs are able to survive within rabbit intervertebral discs (14).

Scaffold selection is critical for cell transplantation, more so than cell culture and cell differentiation capacity. PLGA scaffolds that were safely degraded to water and carbon dioxide, and that had stable physical and chemical properties, a negligible foreign body reaction and no immunogenicity were selected. PLGA scaffolds were also highly porous, allowing for 3-dimensional (3D) growth of cells as well as biological structural plasticity and mechanical strength. Richardson et al (19) reported the presence of NP chondrocytes in all degradable 3D PLGA scaffolds with attached BM-MSCs transformed by NPs. Von Heimburg et al (20) suggested that ideal cell scaffolds should be sufficiently open to allow connectivity and have a directional pore structure (diameter $>120 \mu \mathrm{m}$ ). Cells attached well and were histocompatible with PLA: PGA at ratio of 75:25 (21). This provided mechanical support and area for early transplanted cell growth as well as metabolite exchange capacity due to high porosity (>90\%). PLGA scaffolds made from low-temperature and high-pressure methods met these criteria.

In previous studies, New Zealand rabbits were dissected along the costal margin to the iliac incision with a fixed lateral position, blunting dissection along the internal oblique muscle and paraspinal boundaries, until the paraspinal muscle could be retracted to the front or side of the vertebral body $(13,14)$. This is difficult using a lateral approach and may cause vertebral fractures and strip muscles, as lumbar transverse dissection extending downward toward the side of the front transverses by 3-4 cm, where paraspinal muscles are tightly attached to the transverse, complicating recovery and causing mortality (22). In the present study, an abdominal oblique anterolateral incision was made parallel to the iliac crest with a supine extraperitoneal approach, separating from the abdominal oblique muscle and peritoneum, cutting to the front of the vertebral body from the outside into the peritoneum. This was more likely to expose the intervertebral space with less muscle and soft tissue injury and facilitated post-operative recovery.

Tissue engineering and disc construction using BM-MSCs transformed by NPs are in their infancy and regulatory mechanisms for differentiation of BM-MSCs remain elusive. In addition, the long-term effects and safety of BM-MSCs are currently unknown. Improved biomechanical properties, controlled and slow scaffold degradation, balancing of mechanical strength and optimal tissue formation to maximize the repair of intervertebral discs with tissue engineering approaches are goals of future research.

\section{Acknowledgements}

The authors would like to thank LetPub (www.letpub. com) for linguistic assistance during the preparation of this manuscript. The present study was supported by the National Natural Science Foundation of China (grant nos. 81371998 and 81672200) and the National Key Clinical Specialty Construction Project of China.

\section{References}

1. Hoy D, Brooks P, Blyth F and Buchbinder R: The epidemiology of low back pain. Best Pract Res Clin Rheumatol 24: 769-781, 2010.

2. Takahashi K, Aoki Y and Ohtori S: Resolving discogenic pain. Eur Spine J 17 (Suppl 4): S428-S431, 2008.

3. McMeeken J, Tully E, Stillman B, Nattrass C, Bygott IL and Story I: The experience of back pain in young Australians. Man Ther 6: 213-220, 2001

4. Smith LJ, Nerurkar NL, Choi KS, Harfe BD and Elliott DM: Degeneration and regeneration of the intervertebral disc: Lessons from development. Dis Model Mech 4: 31-41, 2011.

5. Chadderdon RC, Shimer AL, Gilbertson LG and Kang JD: Advances in gene therapy for intervertebral disc degeneration. Spine J 4 (Suppl 6): S341-S347, 2004.

6. Nomura T, Mochida J, Okuma M, Nishimura K and Sakabe K: Nucleus pulposus allograft retards intervertebral disc degeneration. Clin Orthop Relat Res 94-101, 2001.

7. Minguell JJ, Erices A and Conget P: Mesenchymal stem cells. Exp Biol Med (Maywood) 226: 507-520, 2001.

8. Thompson JP, Pearce RH, Schechter MT, Adams ME, Tsang IK and Bishop PB: Preliminary evaluation of a sheme for grading the gross morphology of human intervertebral disc. Spine (Phila $\mathrm{Pa}$ 1976) 15: 411-415, 1990 .

9. Gao Hua, Liu Kun and Yu Zidong: Spectrophotometric Determination of Chondroit in Sulfate with Phlorglucinol. Chin J Biochemical Pharmaceutics 5: 247-248, 2000.

10. Sato M, Asazuma T, Ishihara M, Ishihara M, Kikuchi T, Kikuchi $\mathrm{M}$ and Fujikawa K: An experimental study of the regeneration of the intervertebral disc with an allograft of cultured annulus fibrosus cells using a tissue-engineering method. Spine (Phila Pa 1976) 28: 548-533, 2003.

11. Leung VY, Chan D and Cheung KM: Regeneration of intervertebral disc by mesenchymal stem cells: Potentials, limitations, and future direction. Eur Spine J 15 (Suppl 3): S406-S431, 2006.

12. Richardson SM, Walker RV, Parker S, Rhodes NP, Hunt JA, Freemont AJ and Hoyland JA: Intervertebral disc cell-mediated mesenchymal stem cell differentiation. Stem Cell 24: 707-716, 2006.

13. Li Quan-Xiu, Chen Bo-Hua, Liu Yong and Xu Ling: Construction of in vitro models of human bone marrow-derived mesenchymal stem cells differentiated into nucleus pulposus-like cells in rabbits. J Clin Rehabilitative Tissue Engineering Res 13: 8961-8965, 2009.

14. Wang Yue-Qiu and Chen Bo-Hua: Experimental observation of rabbit intervertebral disc transplantation with human bone marrow mesenchymal stem cells. J Clin Rehabilitative Tissue Engineering Res 14: 4951-4956, 2010.

15. Li Quan-Xiu, Chen Bo-Hua and Cai Yue-Yan: Differentiation of human bone marrow mesenchymal stem cells-induced nucleus pulposus cells under new-style layer coculture. J Clin Rehabilitative Tissue Engineering Res 14: 53-56, 2010.

16. Gu Rui and Chen Bohua: Obsercation of survivial state of exogenous mesenchymal stem cells in rabbit intervertebral disc. Actc Academic Med Qingdao Univ 45: 447-448, 2009.

17. Nerlich AG, Schaaf R, Wälchli B and Boos N: Temporo-spatial distribution of blood vessels in human lumbar intervertebral discs. Eur Spine J 16: 547-555, 2007. 
18. Tse WT, Pendleton JD, Beyer WM, Egalka MC and Guinan EC: Suppression of allogeneic T-cell proliferation by human marrow stromal cells: Implications in transplantation. Transplantation 5: 389-397, 2003.

19. Richardson SM, Curran JM, Chen R, Vaughan-Thomas A Hunt JA, Freemont AJ and Hoyland JA: The differentiation of bone marrow mesenchymal stem cells into chondrocyte-like cells on poly-L-lactic acid (PLLA) scaffolds. BiomaIerials 27: 4069-4078, 2006

20. von Heimburg D, Kuberka M, Rendchen R, Hemmrich K, Rau G and Pallua N: Preadipocyte-loaded collagen scaffolds with enlarged pore size for improved soft tissue engineering. Int J Anif Organs 6: 1064-1076, 2003
21. Hu X, Shen H, Yang F, Bei J and Wang S: Preparation and cell affinity of microtubular orientation-structured PLGA(70/30) blood vessel scaffold. Biomaterials 29: 3128-3136, 2008.

22. Liu J, Wang J and Zhou Y: BMSCs-chitosan hydrogel complex transplantation for treating intervertebral disc degeneration. Zhongguo Xiu Fu Chong Jian Wai Ke Za Zhi 23: 178-182, 2009 (In Chinese). 